\title{
Effect of a Pedometer-Based, 24-Week Walking Intervention on Depression and Acculturative Stress among Migrant Women Workers
}

\author{
Youlim Kim ${ }^{1} \mathbb{D}$, Young-Me Lee ${ }^{2}$, Mikyeong $\mathrm{Cho}^{1}$ and Hyeonkyeong Lee ${ }^{1, *}$ \\ 1 College of Nursing, Yonsei University, 50-1, Yonsei-ro, Seodaemun-gu, Seoul 03722, Korea; \\ goshimak@naver.com (Y.K.); zzomi324@naver.com (M.C.) \\ 2 School of Nursing, DePaul University, Chicago, IL 60640, USA; YLEE23@depaul.edu \\ * Correspondence: hlee39@yuhs.ac; Tel.: +82-2-2228-3373
}

Received: 20 September 2019; Accepted: 7 November 2019; Published: 9 November 2019

check for updates

\begin{abstract}
Little research has examined exercise-based interventions meant to alleviate depressive symptoms among Korean-Chinese migrant women workers living in Korea. Thus, this study evaluated the effectiveness of a 24-week walking program on reducing depressive symptoms and acculturative stress levels in this population. This quasi-experimental sequential walking program was conducted with 132 Korean-Chinese women over a period of 24 weeks. Participants were divided into either a standard treatment group $(n=69)$ or enhanced treatment group $(n=63)$. All participants were instructed to walk using a pedometer. The enhanced treatment group also received mobile text messages designed to encourage walking adherence and improve acculturation. Participants were asked to complete two structured questionnaires, the Centre for Epidemiologic Studies Depression Scale and the Acculturative Stress Scale, to evaluate the intervention programs. At the end of the program, both groups exhibited decreased depression scores, but the decrease in the enhanced treatment group was more significant both at weeks 12 and 24 . Acculturative stress was also found to have decreased at weeks 12 and 24. Our findings show the walking program reduced the depressive symptoms and acculturative stress levels among the Korean-Chinese women in this study. Further studies will be needed to analyze the relationship between walking step count and mental health considering exercise intensity.
\end{abstract}

Keywords: physical activity; physical intervention; health behavior; health promotion; cognitive-behavioral therapy

\section{Introduction}

Depression is a common mental disorder that is indicated by a depressive emotion, loss of interest or pleasure, loss of energy, sleep or appetite disorders, and decreased concentration [1]. More than 300 million people of all ages around the world suffer from depression, and women are more likely than men to have depression [2]. Furthermore, depression remains a substantial mental health problem across migrant populations. Based on data from 25 studies, the prevalence rate among migrants was found to be $15.6 \%$ [3]. Migrant populations are recognized as being at risk for adverse mental health issues due to post-migration stressors and the process of adjusting to the culture and system of their host country [4]. Nevertheless, individuals in these populations often have limited access to mental health services due to barriers such as language, lack of familiarity with mainstream services, and cultural beliefs [5]. Although migrant populations are among the most vulnerable and often need special attention from healthcare professionals, limited research has been done on the effectiveness of interventions to improve mental health outcomes for migrant workers. 
Korean-Chinese (KC) migrants are the largest immigrant group in Korea, with about 680,000 residents accounting for roughly $31 \%$ of the total immigrants in 2017 [6]. Moreover, one study found that $70.4 \%$ of KC women aged 40 years or older [5] worked as housekeepers or in restaurants $[7,8]$. This is significant because an earlier study on acculturative stress and depression among KC migrant workers [9] found that service workers reported higher rates of depression when compared to other types of workers, and women workers had significantly higher rates of depression than male workers. Specifically, KC migrant workers with high levels of acculturative stress were more likely to experience depressive symptoms than those who had low levels of acculturative stress.

Previous research has shown that walking is beneficial in the decrease of depressive symptoms. In a 12-week walking intervention for middle-aged Iranian women [10], an increased number of steps significantly lowered the participants' depression severity and, in another study [11], the more the number of walking steps increased, the more the depression scores decreased. In addition, acculturation has been shown to be associated with an individual's health behaviors [12]. Acculturative stress, which is defined as the losses that arise when adapting or assimilating to a different culture with regard to beliefs, routines, and social roles [13], is also related to adherence to health behaviors. Culturally assimilated migrants have been reported to be more physically active compared to a lower acculturation group [12].

However, achieving the same positive mental health effects with walking seems to be challenging when applied to migrant populations. Migrant populations may place a different level of importance on the benefits of physical activities [14]. Their ability to perform physical activities is often limited by a lack of time, motivation, energy, and financial resources [7,15].

In addition, migrant populations often struggle with low socioeconomic status and isolation from mainstream channels of communication, which can create further barriers to participation in intervention programs promoting health [16]. Therefore, cognitive behavioral strategies focused on engaging members of migrant populations to maintain recommended walking routines need to be incorporated into an intervention program that is culturally adaptive and collectively created by both participants and researchers to meet the needs of migrant populations [17]. This can be done by using a community-based participatory research (CBPR) approach to create health-related interventions. CBPR promotes co-learning and capacity building among all partners and facilitates collaborative, equitable partnerships during the entire research process [18]. As such, the research presented herein is expected to empower $\mathrm{KC}$ migrant communities and encourage positive health outcomes, including the decrease of cardiovascular disease risks [19]. However, the influence of culturally-adaptive CBPR-based interventions on the mental health outcomes of KC migrant women workers living in Korea is currently not well understood. Thus, we aim to examine the efficacy of a culturally-adaptive 24-week home-based walking intervention on reducing acculturative stress levels and depressive symptoms among KC migrant women workers.

\section{Materials and Methods}

This study used a quasi-experimental sequential design and participants were assigned to either the standard treatment (ST) or enhanced treatment (ET) group. A total of 132 middle-aged female KC migrant workers participated. This study was conducted from May 2012 to April 2015; ST data were collected in 2013 and ET data were collected in 2014. The study protocol is described in detail in Cho et al.'s work [17].

\subsection{Participants}

Participants were recruited through posters, word of mouth or the distribution of leaflets at three KC churches, a migrant support center, and two KC markets. The inclusion criteria used for this study selected KC migrant women workers who were between 40 and 65 years of age; had worked full-time during the previous six months; spoke Korean; and had regular access to a mobile phone. 
$\mathrm{KC}$ women who usually engaged in regular exercise (i.e., 30 minutes a day more than three days a week during the past three months) or with medical restrictions in terms of physical activity readiness were excluded. Physical activity readiness was measured using the Physical Activity Readiness Questionnaire, which tests minimum readiness for moderate physical activity programs [18].

All participants in both the ST and ET groups received a pedometer to use during the 24-week walking intervention. During a 12-week adaptation period, the participants received assistance to further promote adherence to the program's walking routine. This was followed by a 12-week maintenance period in which the participants were expected to continue program adherence on their own.

\subsection{Walking Adherence}

Walking adherence was measured twice, at 12 and 24 weeks, and was defined as the average number of steps per day over the previous 12 weeks. The daily number of steps was measured with a pedometer (DIGI-WALKER CW-700/701; Yamax, Japan). Steps less than 300 count were considered invalid data because it meant that the pedometer was either not used or had malfunctioned [20]. Each week needed to have three or more days with valid data of at least 300 steps a day to be counted [21].

\subsection{Depression}

The Korean version of the Center for Epidemiologic Studies Depression Scale [22,23] was used to measure the level of depression experienced by the participants. This assessment tool consists of 20 items used to measure an individual's experience of depression during the previous week. It uses a four-point scale (nearly $=0$ points, sometimes $=1$ point, significantly $=2$ points, and mostly $=3$ points), with scores ranging from 0-60. Higher scores indicate higher levels of depression. Cronbach's $\alpha$ ranged from 0.85 to 0.90 in the original study [18] and Cronbach's $\alpha$ for this study sample was 0.92 .

\subsection{Acculturative Stress}

Acculturative stress was measured with the Acculturative Stress Scale for International Students [24], which was translated into Korean with an established acceptable internal consistency reliability (Cronbach's $\alpha$ of 0.94 ) by Yang [25]. It consists of 36 total items: perceived discrimination (8 items), homesickness (4 items), perceived hate/rejection (5 items), fear (4 items), culture shock ( 3 items), guilt ( 2 items), and miscellaneous (10 items). We only used 35 items, excluding one item related to discrimination based on skin color, as it was not relevant to our study. Each item was rated on a five-point scale ranging from 1 (strongly disagree) to 5 (strongly agree), and higher scores indicated higher levels of acculturative stress. Cronbach's $\alpha$ was 0.92 in this study.

\subsection{Intervention}

\subsubsection{Standard Treatment}

The intervention was developed using the Intervention Mapping method [26] to enhance participants' social-psychological and cognitive competence, as well as to improve their cultural adaptation and exercise adherence. Program orientation and baseline assessment were conducted by trained research assistants. Participants were provided with program manuals, a pedometer, and training in walking and stretching. Based on the guideline by the American College of Sports Medicine [27], in the program orientation, the participants were instructed to walk with moderate intensity to maximize the psychological benefits of exercise. Using this guideline, we encouraged participants to walk one step every two seconds. A trained nurse was consulted when setting the daily step goal. According to the number of steps taken per day, the physical activity of the participants was classified into five levels: sedentary ( $<5000$ steps/day); low active (5000-7499 steps/day); somewhat active (7500-9999 steps/day); active (10,000-12,499 steps/day); and highly active ( $\geq 12,500$ steps/day) [28]. The established goal was to increase the number of steps taken by more than 3000 per day within four 
weeks [29]. This goal was implemented three times; at baseline, week 4 and week 8 . Participants were asked to record their number of daily steps every day for 24 weeks and to report this information to the research team via mobile phone every 2 weeks.

\subsubsection{Enhanced Treatment}

During the 12-week adaptation period, only the ET group received 12 exercise-related motivational text messages and 12 medal images (such as gold, silver, or bronze) to encourage exercise adherence. To improve cultural adaptation, informational illustrations portraying real-life experiences necessary to living in Korea, such as how to order drinks in cafés and how to read dry-cleaning labels, were identified by a needs survey. Those illustrations, which make cultural information easy to understand, were sent to participants' mobile phones once during every 2-week period [17].

\subsection{Data Collection}

The study was approved by the ethics committee from the institution where the research was conducted (No. 2012-0008). Prior to the collection of data, trained research assistants explained the purpose of the study, highlighting the need for participant engagement in research, and then obtained written informed consent. The participants were informed of their right to voluntary participation and that they could stop participation at any point in the study. In order to preserve anonymity, participants' names cannot be identified on any data collection sheets. To help ensure all participants understood the terms of the study, face-to-face data collection was conducted by trained Korean research assistants using structured questionnaires. All data (except for demographic characteristics) were measured at the beginning of the study for a baseline, then again at weeks 12 and 24 .

\subsection{Statistical Analyses}

Data were analyzed using IBM SPSS Statistics 22.0. Descriptive statistics (frequency, means, and standard deviations) were used to describe participants' basic characteristics. Group differences were analyzed using independent $t$-tests and chi-squared tests. The walking steps, depression, and acculturative stress scores of 12 weeks and 24 weeks compared to the baseline were conducted by paired $t$-test. Since missing data appeared at various points in the three measurements, a linear mixed model analysis including the missing data was used to confirm the interaction between time and group. We divided the average number of steps by 1000 at three times (baseline, 12 weeks, and 24 weeks) and used the linear mixed effect analysis as a continuous variable to analyze the effect on depression and acculturative stress. The number of steps was not analyzed according to each step, but according to 1000 steps that were combined into a unit based on previous studies [30,31]. Significance was set at 0.05 for all tests. To assess the influence of the baseline imbalance on attrition [32], a propensity score matching technique was used. We calculated the propensity scores of the "attrition group" and the "retention group" through a logistic regression analysis according to the participants' baseline general characteristics (age, education level, income, marital status, duration of stay, chronic diseases, body mass index [BMI], etc.).

\section{Results}

\subsection{Participants' Characteristics and Prospensity Scores Matching}

We initially recruited $264 \mathrm{KC}$ women to participate in this study; however, 80 did not meet the eligibility criteria and 52 did not wish to participate. Therefore, we were left with 132 participants in total. The recruitment and retention of participants is described in an earlier publication of this research project [19].

Participants' general characteristics and baseline walking steps, score of depression, and acculturative stress are shown in Table 1. A homogeneity test was conducted to compare general characteristics such as age, residence time, education, and income for the ST group and the ET group. 
The average age of the participants was $56.20 \pm 5.46$ years in the ST group and $56.62 \pm 4.69$ years in the ET group $(p=0.639)$. The average duration of the stay in Korea for the participants was $81.41 \pm 49.18$ months in the ST group and $126.44 \pm 77.86$ months in the ET group $(p<0.001)$. The average months of working at their current job was $54.06 \pm 40.40$ in the ST group and $61.08 \pm 57.80$ in the ET group $(p=0.419)$. The average time per day the participants spent working was $11.71 \pm 3.17$ hours in the ST group and $13.24 \pm 4.69$ hours in the ET group $(p=0.030)$. The number of high school graduates was 40 $(58.0 \%)$ in the ST group and $41(65.1 \%)$ in the ET group, and there was no difference between the two groups $(p=0.402)$. The monthly income was $1312.54 \pm 147.87$ in the ST group and $1332.64 \pm 359.31$ dollars in the ET group, and there was no difference between the two groups $(p=0.670)$. Regarding participants' types of jobs, housekeeping accounted for the highest proportion ( $\mathrm{n}=106,80.3 \%)$, followed by restaurant waitressing $(\mathrm{n}=13,9.8 \%)$. Other types of jobs included caregiving, self-employment, and so on $(n=13,9.8 \%)$. The number of participants with no chronic diseases was $63(47.7 \%)$, and there was no difference between the groups $(p=0.745)$.

Table 1. Participants' baseline characteristics.

\begin{tabular}{|c|c|c|c|c|c|c|}
\hline \multirow{2}{*}{ Variable } & Total & ST Group $(n=69)$ & ET Group $(n=63)$ & \multirow[b]{2}{*}{$x^{2}$} & \multirow[b]{2}{*}{$t$} & \multirow[b]{2}{*}{$p$} \\
\hline & n (\%)/M(SD) & $\mathrm{n}(\%) / \mathrm{M}(\mathrm{SD})$ & $\mathrm{n}(\%) / \mathrm{M}(\mathrm{SD})$ & & & \\
\hline Age & $54.60(5.09)$ & $56.20(5.46)$ & $56.62(4.69)$ & & 0.47 & 0.639 \\
\hline Duration of stay (months) & $102.90(68.08)$ & $81.41(49.18)$ & $126.44(77.86)$ & & 4.01 & $<0.001$ \\
\hline Duration of current job (months) & $57.44(49.46)$ & $54.06(40.40)$ & $61.08(57.80)$ & & 0.81 & 0.419 \\
\hline Working time(h/day) & $12.45(4.03)$ & $11.71(3.17)$ & $13.24(4.69)$ & & 2.20 & 0.030 \\
\hline Income (USD/month) & $\begin{array}{l}1322.13 \\
(269.36)\end{array}$ & $1312.54(147.87)$ & $1332.64(359.31)$ & & 0.43 & 0.670 \\
\hline \multicolumn{7}{|l|}{ Education } \\
\hline$\geq$ High school & $81(61.8)$ & $40(58.0)$ & $41(65.1)$ & 0.702 & & 0.402 \\
\hline \multicolumn{7}{|l|}{ Type of job } \\
\hline Housekeeper & $106(80.3)$ & $58(84.1)$ & $48(76.2)$ & 1.443 & & 0.486 \\
\hline Waitress & $13(9.8)$ & $5(7.2)$ & $8(12.7)$ & & & \\
\hline Others & $13(9.8)$ & $6(8.7)$ & $7(11.1)$ & & & \\
\hline \multicolumn{7}{|l|}{ Chronic disease } \\
\hline None & $63(47.7)$ & $32(46.4)$ & $31(49.2)$ & 0.106 & & 0.745 \\
\hline Walking(steps/day) & $9,193(3212)$ & $8,728(2,977)$ & $9,701(3,875)$ & & 1.65 & 0.102 \\
\hline Depression & $13.02(8.68)$ & $13.72(7.12)$ & $14.35(10.17)$ & & 0.41 & 0.681 \\
\hline Acculturative stress & $70.42(21.37)$ & $70.33(19.10)$ & $70.51(23.77)$ & & 0.05 & 0.963 \\
\hline
\end{tabular}

Of all the participants, 84 out of $132 \mathrm{KC}$ women completed the 24-week follow-up test (overall attrition rate $=36.4 \%$ ) Therefore, propensity score matching (PSM) analysis was conducted to increase the validity of the intervention by confirming the bias of the retention group. Before matching, a total of 48 participants were in the attrition group, while 84 participants were in the retention group. The PS was calculated using general characteristics variables, and the PS of the retention group was distributed from 0.4 to 1.0, with an average score of 0.63 . The PS of the attrition group was distributed between 0.3 and 0.8 , with an average score of 0.61 . After one-to-one matching according to the PS score, 46 dropouts were paired with 46 retainers. The PS average of the retention group was 0.62 and the PS average of the attrition group was 0.60 , and the distribution was similar between the two groups.

The PS distribution of participants before and after matching and after controlling for general characteristic variables, such as age, duration of stay, working time, income, and education level by covariates, is shown in Figure 1. 

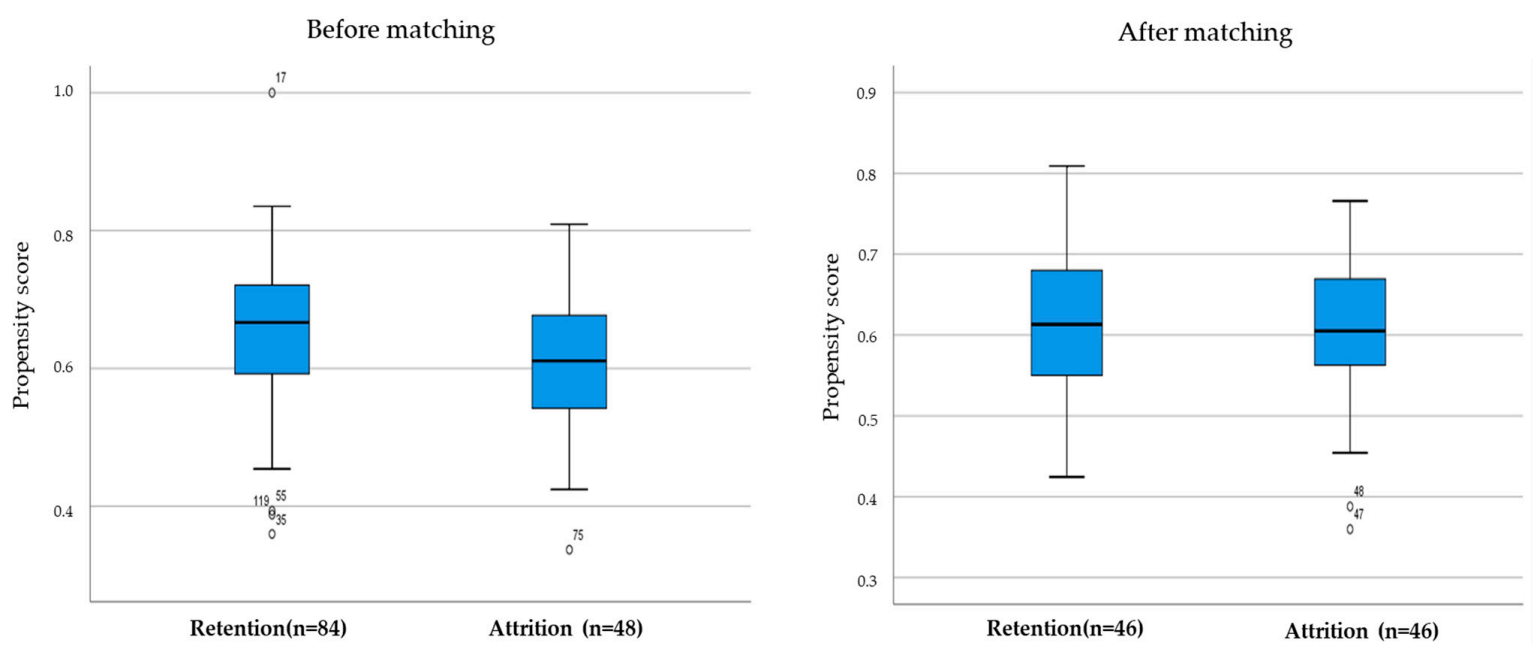

Figure 1. Comparison of propensity score retention and attrition.

\subsection{Walking Adherence, Depression, and Acculturative Stress}

Figure 2 shows the walking adherence, depression, and acculturative stress from the baseline to weeks 12 and 24 in both groups. The comparison between groups was based on the baseline, 12-week, and 24-week average scores. Compared to the baseline, the number of walking steps significantly increased in both the ST and the ET groups at week 12 (ST: $\mathrm{t}=7.473, p<0.001 \mathrm{ET}: \mathrm{t}=5.649, p<0.001$ and at week 24 (ST: $\mathrm{t}=7.668, p<0.001 \mathrm{ET}: \mathrm{t}=3.252, p=0.003$ ). The participants' depression significantly decreased at 12 and 24 weeks compared to the baseline in the ET group (at week 12: $\mathrm{t}=-3.244, p=0.002$, at week 24: $\mathrm{t}=-3.368, p=0.002$ ). Similarly, participants' acculturative stress significantly decreased at 12 and 24 weeks compared to the baseline in the ET group (at week 12: $\mathrm{t}=-2.393, p=0.021$, at week 24: $\mathrm{t}=-2.464, p=0.018)$.

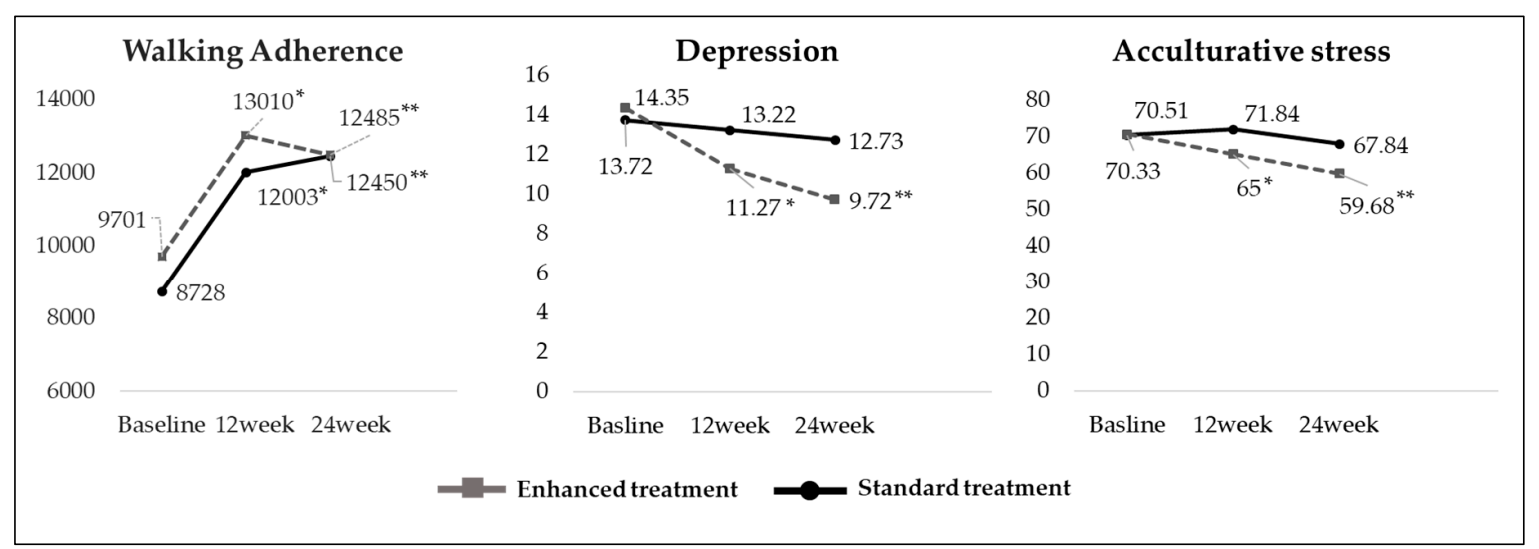

Figure 2. Comparison of walking adherence, depression, and acculturative stress levels between enhanced treatment (ET) and standard treatment (ST) groups over the 24-week study. ${ }^{*} p<0.05$ : difference between baseline and 12 week, ${ }^{* *} p<0.05$ : difference between baseline and 24 week.

\subsection{Changes in Depression and Acculturative Stress}

Table 2 reflects the linear mixed effects models for the depression and acculturative stress outcomes after adjusting for baseline characteristics of age, monthly income (USD), type of job, duration of stay, education, working time per day, and chronic disease. In the linear mixed model analysis, the number of walking steps-measured three times-was included, and as the daily steps increased by 1000 steps, all participants showed a significant reduction in depressive symptoms and acculturative stress. In addition, the analysis showed a significant interaction effect of group and time for depression at weeks 12 and 24, compared to the baseline. Similarly, there was a significant effect of walking on 
acculturative stress reduction for all participants. A significant interaction effect between group and time for acculturative stress was shown at weeks 12 and 24 compared to the baseline.

Table 2. Changes in depression and acculturative stress scores over time.

\begin{tabular}{lcccc}
\hline \multicolumn{1}{c}{ Outcome } & B & SE & t & $p$ \\
\hline Depression & & & & \\
Steps/day (per 1000 steps) & -0.308 & 0.121 & -2.545 & $\mathbf{0 . 0 1 2}$ \\
ET (reference: ST) & 0.701 & 1.551 & 0.452 & 0.652 \\
Week 12 (reference: Baseline) & 0.493 & 1.087 & 0.453 & 0.651 \\
Week 24 (reference: Baseline) & -0.124 & 1.041 & -0.119 & 0.906 \\
ET*Week 12 & -3.149 & 1.444 & -2.180 & $\mathbf{0 . 0 3 1}$ \\
ET*Week 24 & -3.104 & 1.380 & -2.249 & $\mathbf{0 . 0 2 7}$ \\
Acculturative stress & & & & \\
Steps/day (per 1000 steps) & -0.748 & 0.330 & -2.266 & $\mathbf{0 . 0 2 4}$ \\
ET (reference: ST) & -1.255 & 3.894 & -0.322 & 0.748 \\
Week 12 (reference: Baseline) & 4.119 & 3.132 & 1.315 & 0.190 \\
Week 24 (reference: Baseline) & 1.661 & 2.728 & 0.609 & 0.543 \\
ET*Week 12 & -8.679 & 4.202 & -2.066 & $\mathbf{0 . 0 4 0}$ \\
ET*Week 24 & -7.178 & 3.612 & -1.987 & $\mathbf{0 . 0 4 9}$ \\
\hline
\end{tabular}

The model is adjusted for the baseline characteristics of age, monthly income, type of job, duration of stay, education, working time per day, and chronic disease $(p<0.05)$; Bold indicates statistically significant $p$-values $(p<0.05)$; * indicates interaction of Group and Time.

\section{Discussion}

This study was designed to evaluate the effect of a pedometer-based 24-week walking program on depression and acculturative stress among KC migrant women workers. While both groups had significantly increased their walking steps at 12 or 24 weeks compared to the baseline, depression and acculturative stress decreased in the ET at weeks 12 and 24 due to significant interactions between time and group. This suggests that the intervention enhanced by socio-cognitive psychological factors that was applied to the ET was effective.

This study demonstrated the effectiveness of a home-based walking intervention with the use of a pedometer to enhance cultural adaptation and decrease the levels of depression and acculturative stress experienced by middle-aged women KC migrant workers. Given the continuous increase in the number of KC migrant workers coming to Korea, the need to better understand how exercise interventions improve their mental health is important and timely. This study is among the first to examine the effects of a walking-based intervention, which has been shown to be a protective factor against depression [33], on commonly experienced symptoms, such as depression and acculturative stress, among KC migrant workers living in Korea. As such, these findings make a positive contribution to the existing body of literature.

Compared to the baseline, the walking-based intervention showed a positive effect on the reduction of depressive symptoms at 24 weeks, which is in line with findings for similar interventions [21,33-35]. Notably, we observed a sustained reduction in depressive symptoms for the ET group only. These results suggest that participants who have an additional cultural adaptation intervention paired with walking regularly experience fewer depressive symptoms and show less acculturative stress than those who only walk.

In our study, the number of steps a day was measured using 1000 steps as a unit. Gilson et al. [30] as well as Butler et al. [31] encouraged participants to walk an additional 1000 steps daily to promote physical activity in the workplace. Walking an additional 1000 steps per day is also associated with a lower BMI [36] and improvement in self-reported depressive symptoms [37]. Likewise, we found that when the number of steps was increased by 1000 per day, participants' depressive symptoms decreased. Therefore, we recommend a progressive increase of at least 1000 steps per day for people who walk fewer than 10,000 steps in order to promote the health benefits associated with walking. 
Concerning acculturative stress, the significant reduction in the earlier phase of the study was maintained through to week 24 . KC migrant women experience a variety of adaptation challenges and stressful life events in the workplace and at home. It has been reported that members of migrant populations commonly encounter difficulties adjusting to numerous changes in their lives [38]. The high levels of acculturative stress that accompany this process of adaptation may result in increased mental health concerns. Therefore, in addition to the provision of walking intervention, a supportive environment at work and in the community is needed to help migrant populations adapt to their new culture.

CBPR has been used as an effective way of approaching health interventions in migrant populations $[39,40]$. As such, the CBPR approach was adopted by this study particularly to address the challenges of delivering culturally-appropriate walking interventions. For example, the study participants were asked to name the walking program and prioritize topics that would be most applicable to KC women. This process was appropriate to allow them to have ownership of the intervention as well as the commitment to complete it. However, we still had a considerable attrition rate of $36.4 \%$, although attrition in some form is a nearly universal reality in longitudinal research with human participants. In this study, no different characteristics were identified between the retention group and attrition group; however, strategies to decease the attrition rate should be considered in future studies, as high attrition can threaten the external validity of an intervention by producing a final sample that is not representative of the population sample [41].

This study included several limitations. Primarily, it was difficult to design a randomized controlled trial (RCT) study in a KC migrant community. As mentioned in an earlier related study [19], it is possible that the participants in the intervention group sometimes interact with participants in the control group, which creates bias; however, this is nearly impossible to avoid due to social interactions among individuals in the same residential area [42]. In this study, the number of steps also increased significantly in the ST group provided with the same pedometer as the ET group. According to the systematic literature review of Bravata et al. [43], the use of pedometers tends to increase a person's motivation for walking. Similarly, in a study of low socioeconomic groups [44], physical activity level was found to increase significantly in the control group, which only received basic health services. Therefore, it is assumed that providing basic intervention to individuals with low awareness of health care services has motivated them to adhere to health promotive behavior. In addition, since the participants are engaged in jobs that require high occupational physical activity - such as housekeeping and waitressing-one should be careful in extrapolating the findings to those who require low occupational physical activity, such as office workers. Since this was an intervention study of home-based walking within a community setting, the influence of exogenous variables may exist due to limited control over participants. Also, recruitment and retention may also have been limited because $\mathrm{KC}$ migrant workers were difficult to reach out to due to a limited budget and time schedule. Migrant populations are often considered hard-to-reach groups, and more effective strategies may be needed to overcome these difficulties. Such strategies may include using pre-existing community networks and building trust within the migrant population [16]. Further research is needed to determine practical solutions to better engage the KC migrant community.

Migration is not a cause of mental health issues in and of itself, but it may lead to individuals being exposed to many stressors associated with settling into a new host country. These circumstances contribute to an increase in acculturative stress levels. Those increased levels are, in turn, related to the increase of depressive symptoms among members of migrant populations. Thus, nurses and community leaders need to be aware of these mental health issues and work to prevent and manage the adverse effects associated with migration. With migration increasing globally, health professionals working with migrant populations should offer regular walking (based on a cognitive behavioral approach) as a way to promote positive mental health. 


\section{Conclusions}

This study demonstrated that a 24-week walking intervention was suitable for decreasing depressive symptoms and levels of acculturative stress in middle-aged KC migrant women workers who were not currently prioritizing their health. Culturally adaptive triggers to motivate the KC workers to continue exercising were effective, but they may need to be updated further depending on the participants' needs. Depression is a commonly experienced mental health concern among migrant populations worldwide, and the walking intervention presented in this study-which was further enhanced by social-cognitive elements-may be useful for groups of other ethnicities as well. In this study, walking measurements relied on participants' self-reporting of the number of steps. Further studies are needed to examine the influence of moderate intensity walking on mental health using objective measures of walking.

Author Contributions: Y.K. performed the analysis and drafted the manuscript. H.L. conceived study, supervised the project, and revised the manuscript critically. Y.-M.L. and M.C. discussed the results and critiqued the manuscript. All authors reviewed and approved the final manuscript.

Funding: This research was supported by a National Research Foundation of Korea (NRF-2012R1A1A3019009, NRF-2017R1A2B4008671) with the fund of the Korean government (MSIP; Ministry of Science, ICT and Future Planning).

Acknowledgments: We want to thank Chang-Gi Park and Ki Jun Song who advised on statistical analysis.

Conflicts of Interest: The authors declare no conflicts of interest.

\section{References}

1. World Health Organization. Depression and Other Common Mental Disorders. Available online: https: //apps.who.int/iris/bitstream/handle/10665/254610/WHO-MSD-MER-2017.2-eng.pdf?sequence=1 (accessed on 8 October 2019).

2. World Health Organization. Depression. Available online: https:/www.who.int/news-room/fac.sheets/ detail/depression (accessed on 10 October 2019).

3. Foo, S.; Tam, W.; Ho, C.; Tran, B.; Nguyen, L.; McIntyre, R.; Ho, R. Prevalence of depression among migrants: A systematic review and meta-analysis. Int. J. Environ. Res. Public Health 2018, 15, 1986. [CrossRef] [PubMed]

4. Bas-Sarmiento, P.; Saucedo-Moreno, M.; Fernández-Gutiérrez, M.; Poza-Méndez, M. Mental health in immigrants versus native population: A systematic review of the literature. Arch. Psychiatr. Nurs. 2017, 31, 111-121. [CrossRef] [PubMed]

5. Salami, B.; Salma, J.; Hegadoren, K. Access and utilization of mental health services for immigrants and refugees: Perspectives of immigrant service providers. Int. J. Ment. Health Nurs. 2019, 28, 152-161. [CrossRef] [PubMed]

6. Statistics Korea. Korea Immigration Service Statistics. Available online: http://kosis.kr/statisticsList/ statisticsListIndex.do?menuId=M_01_01\&vwcd=MT_ZTITLE\&parmTabId=M_01_01\#SelectStatsBoxDiv (accessed on 20 May 2019).

7. Lee, H.; Chae, D.; Cho, S.; Kim, J.; Yoo, R. Influence of a community-based stretching intervention on the health outcomes among Korean-Chinese female migrant workers in South Korea: A randomized prospective trial. Jpn. J. Nurs. Sci. 2017, 14, 277-287. [CrossRef] [PubMed]

8. Lee, C.; Choi, S. Employment Situations of Chinese of Korean Ethnicity by Residential Status in South Korea; No. 2014-09; IOM Migration Research and Training Centre: Goyang-si, Gyeonggi-do, Korea, 2014.

9. Lee, H.; Ahn, H.; Miller, A.; Park, C.; Kim, S. Acculturative stress, work-related psychosocial factors and depression in Korean-Chinese migrant workers in Korea. J. Occup. Health 2012, 54, 206-214. [CrossRef] [PubMed]

10. Abedi, P.; Nikkhah, P.; Najar, S. Effect of pedometer-based walking on depression, anxiety and insomnia among postmenopausal women. Climacteric 2015, 18, 841-845. [CrossRef] [PubMed]

11. Yuenyongchaiwat, K. Effects of 10,000 steps a day on physical and mental health in overweight participants in a community setting: A preliminary study. Braz. J. Phys. Ther. 2016, 20, 367-373. [CrossRef] [PubMed] 
12. Delavari, M.; Sønderlund, A.; Mellor, D.; Mohebbi, M.; Swinburn, B. Migration, acculturation and environment: Determinants of obesity among Iranian migrants in Australia. Int. J. Environ. Res. Public Health 2015, 12, 1083-1098. [CrossRef] [PubMed]

13. Hovey, J.D. Acculturative stress, depression, and suicidal ideation in Mexican immigrants. Cult. Divers. Ethn. Minor Psychol. 2000, 6, 134-151. [CrossRef] [PubMed]

14. Gidengil, E.; Stolle, D. The role of social networks in immigrant women's political incorporation. Int. Migr. Rev. 2009, 43, 727-763. [CrossRef]

15. Juarbe, T.; Turok, X.P.; Pérez-Stable, E.J. Perceived benefits and barriers to physical activity among older Latina women. West. J. Nurs. Res. 2002, 24, 868-886. [CrossRef] [PubMed]

16. Shaghaghi, A.; Bhopal, R.; Sheikh, A. Approaches to recruiting 'hard-to-reach' populations into re-search: A review of the literature. Health Promot. Perspect. 2011, 1, 86-94. [PubMed]

17. Cho, S.; Lee, H.; Kim, J.H.; Lee, M.; Lee, Y.M. Culturally adaptive walking intervention for Korean-Chinese female migrant workers. Res. Theor. Nurs. Pract. 2017, 31, 179-196. [CrossRef] [PubMed]

18. Thomas, S.; Reading, J.; Shephard, R.J. Revision of the physical activity readiness questionnaire (PAR-Q). Can. J. Sport. Sci. 1992, 17, 338-345. [PubMed]

19. Lee, H.; Cho, S.; Wilbur, J.; Kim, J.; Park, C.-G.; Lee, Y.-M.; Lee, H. Effects of culturally adaptive walking intervention on cardiovascular disease risks for middle-aged Korean-Chinese female migrant workers. Arch. Environ. Occup. Health 2017, 72, 317-327. [CrossRef] [PubMed]

20. Cupples, M.; Dean, A.; Tully, M.A.; Taggart, M.; McCorkell, G.; O’Neill, S.; Coates, V. Using pedometer step-count goals to promote physical activity in cardiac rehabilitation: A feasibility study of a controlled trial. Int. J. Phys. Med. Rehabil. 2013, 1, 157.

21. Wilbur, J.; Zenk, S.; Wang, E.; Oh, A.; McDevitt, J.; Block, D.; McNeil, S.; Ju, S. Neighborhood characteristics, adherence to walking, and depressive symptoms in midlife African American women. J. Women Health 2009, 18, 1201-1210. [CrossRef] [PubMed]

22. Radloff, L. The CES-D scale: A self-report depression scale for research in the general population. Appl. Psychol. Meas. 1977, 1, 385-401. [CrossRef]

23. Chon, K.; Lee, M. Development of Korean Version of CES-D: A Preliminary Study. Korean Psychol. Assoc. 1992, 1, 437-445.

24. Sandhu, D.; Asrabadi, B. Development of an acculturative stress scale for international students: Preliminary findings. Psychol. Rep. 1994, 75, 435-448. [CrossRef] [PubMed]

25. Yang, O.; Kim, Y. A study on the effects of depression among foreign wives through marriage migration. Ment. Health Soc. Work 2007, 26, 79-110.

26. Bartholomew, L.K.; Parcel, G.S.; Kok, G. Intervention Mapping: A process for designing theory and evidence-based health education programs. Health Educ. Behav. 1998, 25, 545-563. [CrossRef] [PubMed]

27. Thompson, W.R.; Gordon, N.F.; Pescatello, L.S. ACSM's Guidelines for Exercise testing and Prescription, 6th ed.; Lippincott: Philadelphia, PA, USA, 2000.

28. Tudor-Locke, C.; Bassett, D. How many steps/day are enough? Sports Med. 2004, 34, 1-8. [CrossRef] [PubMed]

29. Tudor-Locke, C.; Schuna, J.M., Jr. Steps to preventing type 2 diabetes: Exercise, walk more, or sit less? Front. Endocrinol. 2012, 3, 142. [CrossRef] [PubMed]

30. Gilson, N.D.; Puig-Ribera, A.; McKenna, J.; Brown, W.J.; Burton, N.W.; Cooke, C.B. Do walking strategies to increase physical activity reduce reported sitting in workplaces: A randomized control trial. Int. J. Behav. Nutr. Phys. Act. 2009, 6, 43. [CrossRef] [PubMed]

31. Butler, C.E.; Clark, B.R.; Burlis, T.L.; Castillo, J.C.; Racette, S.B. Physical activity for campus employees: A university worksite wellness program. J. Phys. Act. Health 2015, 12, 470. [CrossRef] [PubMed]

32. Berger, V. The reverse propensity score to detect selection bias and correct for baseline imbalances. Stat. Med. 2005, 24, 2777-2787. [CrossRef] [PubMed]

33. Torres, E.R.; Sampselle, C.M.; Neighbors, H.W.; Ronis, D.L.; Gretebeck, K.A. Depressive symptoms and walking in African-Americans. Public Health Nurs. 2015, 32, 381-387. [CrossRef] [PubMed]

34. Gawande, R.; To, M.N.; Pine, E.; Griswold, T.; Creedon, T.B.; Brunel, A.; Lozada, A.; Loucks, E.B.; Schuman-Olivier, Z. Mindfulness training enhances self-regulation and facilitates health behavior change for primary care patients: A randomized controlled trial. J. Gen. Intern. Med. 2019, 34, 293-302. [CrossRef] [PubMed] 
35. Kim, Y.; O'Sullivan, D.; Shin, S. Can 24 weeks strength training reduce feelings of depression and increase neurotransmitter in elderly females? Exp. Gerontol. 2019, 115, 62-68. [CrossRef] [PubMed]

36. Dwyer, T.; Ponsonby, A.L.; Ukoumunne, O.C.; Pezic, A.; Venn, A.; Dunstan, D.; Barr, E.; Blair, S.; Cochrane, J.; Zimmet, P.; et al. Association of change in daily step count over five years with insulin sensitivity and adiposity: Population based cohort study. BMJ 2011, 342, 7249. [CrossRef] [PubMed]

37. Kaleth, A.; Slaven, J.; Ang, D. Does increasing steps per day predict improvement in physical function and pain interference in adults with fibromyalgia? Arthrit. Care Res. 2014, 66, 1887-1894. [CrossRef] [PubMed]

38. Rosano, A.; Dauvrin, M.; Buttigieg, S.C.; Ronda, E.; Tafforeau, J.; Dias, S. Migrant's access to preventive health services in five EU countries. BMC Health Serv. Res. 2017, 17, 588. [CrossRef] [PubMed]

39. Wieland, M.L.; Weis, J.A.; Yawn, B.P.; Sullivan, S.M.; Millington, K.L.; Smith, C.M.; Bertram, S.; Nigon, J.A.; Sia, I.G. Perceptions of tuberculosis among immigrants and refugees at an adult education center: A community-based participatory research approach. J. Immigr. Minor Health 2012, 14, 14-22. [CrossRef] [PubMed]

40. Martinez, I.L.; Carter-Pokras, O. Assessing health concerns and barriers in a heterogeneous Latino community. J. Health Care Poor Underserved 2006, 17, 899-909. [CrossRef] [PubMed]

41. Goodman, J.; Blum, T. Assessing the non-random sampling effects of subject attrition in longitudinal research. J. Manag. 1996, 22, 627-652. [CrossRef]

42. Huang, L.; Lv, J.; Chen, N.; Liu, Y. Recruitment of older adults into randomized controlled trials: Issues and lessons learned from two community-based exercise interventions in Shanghai. J. Sports Health Sci. 2016, 5, 308-314. [CrossRef] [PubMed]

43. Bravata, D.M.; Smith-Spangler, C.; Sundaram, V.; Gienger, A.L.; Lin, N.; Lewis, R.; Stave, C.D.; Olkin, I.; Sirard, J.R.J.J. Using pedometers to increase physical activity and improve health: A systematic review. JAMA 2007, 298, 2296-2304. [CrossRef] [PubMed]

44. Eom, S.O.; Lee, I.J.J. The Effect of Health Coaching Programs on Self-Efficacy, Health Behaviors, and Quality of Life in Hypertensive People Living in Poverty. J. Korean Acad. Nurs. 2017, 47, 380-391. [CrossRef] [PubMed]

(C) 2019 by the authors. Licensee MDPI, Basel, Switzerland. This article is an open access article distributed under the terms and conditions of the Creative Commons Attribution (CC BY) license (http://creativecommons.org/licenses/by/4.0/). 